\title{
METHODS TO IMPROVE LIGNIN'S REACTIVITY AS A PHENOL SUBSTITUTE AND AS REPLACEMENT FOR OTHER PHENOLIC COMPOUNDS: A BRIEF REVIEW
}

\author{
Lihong Hu, ${ }^{\mathrm{a}, \mathrm{b}, \mathrm{c}}$ Hui Pan, ${ }^{\mathrm{b}}$ Yonghong Zhou, ${ }^{\mathrm{a}}{ }^{*}$ and Meng Zhang ${ }^{\mathrm{a}}$ \\ Lignin is readily available as a by-product from the pulp and paper \\ industry. It is considered to be a promising substitute for phenol in \\ phenol-formaldehyde (PF) resin synthesis, given the increasing concerns \\ of the shortage of fossil resources and the environmental impact from \\ petroleum-based products. One hurdle that prevents the commercial \\ utilization of lignin is its low reactivity due to its chemical structure. Many \\ efforts have been made to improve its reactivity by modification and/or \\ depolymerization of lignin molecules. Methylolation and phenolation are \\ the two most studied modification approaches aimed at introducing \\ reactive functional groups to lignin molecules. Modified lignin from these \\ two methods could partially replace phenol in PF resin synthesis. \\ Demethylation of lignin could effectively increase the reactivity of lignin \\ by forming catechol moieties in the lignin macromolecule. Other \\ methods, including reduction, oxidation, and hydrolysis, have also been \\ studied to improve the reactivity of lignin as well as to produce phenolic \\ compounds from lignin. Most current methods of lignin modification are \\ not economically attractive. One can expect that efforts will be continued, \\ aimed at improving the utilization of lignin for value-added products.
}

Keywords: Demethylation; Methylolation; Phenolation; Hydrolysis; Phenolic resin

Contact information: a: Institute of Chemical Industry of Forestry Products, CAF; National Engineering Lab. for Biomass Chemical Utilization; Key Lab. on Forest Chemical Engineering, SFA, Key Lab. of Biomass Energy and Material, Jiangsu Province; Nanjing 210042, P.R.China; b: Calhoun Research Station, Louisiana State University Agricultural Center, 321Hwy 80E, Calhoun, LA 71225 USA; c: Institute of Forest New Technology, CAF, Beijing 100091, P.R.China, *corresponding author: yhzhou1966@yahoo.com.cn

\section{INTRODUCTION}

Lignocellulosic biomass is the world's most abundant renewable material and has long been recognized as a potential feedstock to produce chemicals. As one of the three main components of lignocellulosic material, lignin is among the most abundant biopolymers on earth, second only to cellulose. It is estimated that the planet currently contains $3 \times 10^{11}$ metric tons of lignin with an annual biosynthetic rate of approximately $2 \times 10^{10}$ tons (Argyropoulos et al. 1997). There are large quantities of technical lignin (mainly as kraft lignin and lignosulfonate) generated from the paper making industry every year. However, as a low value compound, almost all lignin is burned to generate energy and recover chemicals. Less than $5 \%$ of the world's lignin supply has been used for other purposes, such as materials for automotive brakes, wood panel products, 
phenolic resins, biodispersants, polyurethane foams, phenolic foams, epoxy resins, and surfactants (Gosselink et al. 2004; Lora et al. 2002; Lee et al. 2002).

Lignin consists of three phenylpropanoid monomers, namely coniferyl, sinapyl, and $p$-coumaryl alcohol. These monomers are interconnected by a multitude of inter-unit bonds that include several types of ethers (e.g., $\beta$-O-4, $\alpha-\mathrm{O}-4$, and 4-O-5) and carboncarbon linkages (Mansouri et al. 2006). Interest in lignin as an alternative to replace phenol in PF resin has been growing steadily over the last decade. Several studies investigated the method of directly reacting lignin with phenol and formaldehyde to form prepolymers. In such applications, lignin served both as a filler and a phenol substitute. Direct use of lignin in PF resins required long pressing time and high pressing temperature due to its extremely low reactivity. Therefore, unmodified lignin is usually not commercially attractive for such applications.

Many efforts have been made to improve the reactivity of lignin as the substitute for phenol in PF resin synthesis. One category of methods is to modify the chemical structure of lignin to increase its potential reactive sites toward formaldehyde. The most studied methods in this category are methylolation (hydroxymethylation) and phenoltion, which introduce reactive functional groups to lignin molecules. On the other hand, demethylation could modify lignin to form catechol moieties in lignin macromolecule and thus increase its reactivity. Other methods, including reduction, oxidation, and hydrolysis, have also been studied to improve the reactivity of lignin as well as to produce phenolic compounds from lignin. The purpose of this brief review is to summarize the useful information of such chemical modification and/or depolymerization methods of lignin for the optimum utilization of lignin as phenol substitute in PF resin synthesis and other valuable phenolic compounds.

\section{DEMETHYLATION}

The quality of lignin-based PF adhesives seldom exceeds that of conventional PF adhesives due to the existence of the ether linkages contained within the structure of lignin, which have occupied most of the reactive sites on lignin molecules. In other words, the lignin monomer has far fewer reactive sites than the phenol ring itself. It is estimated that there are only 0.3 reactive aromatic sites available for the formaldehyde condensation for every nine-carbon unit of kraft lignin, which is only $1 / 10$ that of a phenol molecule (Pizzi et al. 1989; Marton et al.1996). It is also known that more than half of the potentially reactive aromatic hydroxyl groups in kraft lignin are blocked by methyl groups. If such methyl groups could be removed, then the resulting material would have much more free phenolic groups and therefore be more reactive (Okamoto et al. 1996).

Sulfur-mediated demethylation has been studied for a long period of time. It has been successfully scaled up to an industrial level as a by-product of dimethyl sulfoxide production. In a nine-carbon unit of lignin, the ortho positions to the aromatic hydroxyl are substituted by one or two methoxyl groups $\left(-\mathrm{OCH}_{3}\right)$, and therefore the reactivity of the free hydroxyl group is hindered. Sulfur-mediated demethylation could remove one or both of the methyl groups ortho to the phenolic hydroxyl group, and the subsequent 
material is expected to have higher reactivity. Wu et al. (2001) studied the sulfurmediated demethylation of wheat straw soda lignin. The results showed that after demethylation, the content of methoxyl groups decreased from $10.39 \%$ to $6.09 \%$, while the contents of phenolic hydroxyls and carboxyl groups increased from $2.98 \%$ and $5.51 \%$ to $4.58 \%$ and $7.10 \%$, respectively. With up to $60 \%$ substitution of demethylated lignin to phenol in the synthesis of lignin-based phenol formaldehyde (LPF) adhesive, the obtained LPF adhesive was able to meet the requirements of the Chinese national standard for first-class plywood. An et al. (1995) used the same method as described above to demethylate kraft lignin for the synthesis of resol-type PF resin. The brief synthesis procedures were as follows: demethylated lignin was first dissolved in an alkaline solution, then formaldehyde was added to the solution at a desired amount. First-stage LPF resol resins were synthesized at pre-set temperature and time. The resulting LPF resin was applied as plywood adhesive and had comparable pressing time as commercial PF resin. It was also reported that it was possible to substitute phenol completely with demethylated lignin and obtain an acceptable plywood adhesive. In addition, the cost of demethylated lignin was about half that of the same amount of phenol. Campion et al. (1998) reported that treatment of radiata pine kraft and kraft-oxygen pulps with sodium ethanethiolate (NaSEt) could reduce the methoxyl content of the pulps. The reaction was conducted in dimethylformamide solution at $153{ }^{\circ} \mathrm{C}$ under $\mathrm{N}_{2}$ atmosphere. The amount of lignin removed in the subsequent oxygen treatment increased as the extent of demethylation increased.

Other demethylation methods also have been reported, but they have rarely attracted attention. For instance, amine hydrohalide salt could demethylate lignin without substantial degradation of the lignin polymer backbone, and result in a water-insoluble, highly reactive lignin material and methyl halide (Yang et al. 1981).

\section{METHYLOLATION/HYDROXYMETHYLATION}

The goal of lignin methylolation, also called hydroxymethylation, is to introduce hydroxymethyl $\left(-\mathrm{CH}_{2} \mathrm{OH}\right)$ to lignin molecules. Such reactions are normally carried out in an alkaline medium with formaldehyde. Methylolated lignin can be directly incorporated in PF resol resin as wood adhesives at different percentage for use in phenol/formaldehyde resin synthesis.

Zhao and co-workers (1994) studied the hydroxymethylation of pine kraft lignin. The results showed that about 0.36 mole of $-\mathrm{CH}_{2} \mathrm{OH}$ per $\mathrm{C}_{9}$ unit was introduced into the lignin molecule. About 0.33 mole was introduced into a $\mathrm{C}_{5}$ unit of guaiacyl moieties via the Lederer-Manasse reaction. Adhesive made from 50/50 (w/w) blending of hydroxymethylated kraft lignin and PF resin could reach a bond strength of about 65 psi in a laboratory test. Malutan et al. (2008) studied the hydroxymethylation of three types of alkaline lignin. The Lederer-Manasse reaction was the dominant reaction during the methylolation, where hydroxymethyl groups were incorporated onto the aromatic rings of lignin. Undesirable side reactions could occur, including the Cannizzarro reaction, in which formaldehyde reacted with itself, and the Tollens reaction, in which the lignin side chains were substituted by aliphatic methylol groups. 
$\mathrm{Mu}$ et al. (2009) used hydroxymethylated lignin to substitute $40 \%$ (wt) phenol in $\mathrm{PF}$ resin synthesis and obtained a PF resin with low free formaldehyde. The bonding strength of the resulting resin for plywood met the requirements for a type I plywood adhesive (GB/T 9846-2004). Lin et al. (2010) found that there was little difference in properties between the PF resins with partial substitution of phenol by hydroxymethylated lignin and un-modified lignin if the amount of phenol substitution was no more than $30 \%$. However, when the substitution was more than $50 \%$, the resulting PF resin with hydroxymethylated lignin yielded better strength and thermostability than those with unmodified lignin. Nevertheless, the use of hydroxymethylated lignin resulted in a higher content of residual formaldehyde and a lower storage stability than those using unmodified lignin. Methylolated ammonium lignosulfonate was also used as a phenol substitute in PF resin synthesis (Alonso et al. 2004). When the molar ratios of sodium hydroxide to phenol-methylolated ammonium lignosulfonate (S/PL) and formaldehyde to phenolmethylolated ammonium lignosulfonate (F/PL) were 0.6 and 2.5 , respectively, 35\%(wt) of the phenol could be replaced by methylolated ammonium lignosulfonate. The formulated resol resin exhibited similar characteristics to those of commercial PF resol resin.

Lignin obtained from eucalyptus wood by acetic acid pulping was methylolated (Vázquez et al. 1997). Results from a formaldehyde reactivity test and the ${ }^{13} \mathrm{C} N M R$ spectra of the lignin samples before and after methylolation indicated increased reactivity of methylolated lignin. LPF resin modified with methylolated lignin at phenol replacement levels of 20 to $40 \%$ showed similar properties to those of commercial PF resin.

\section{PHENOLATION/PHENOLYSIS}

Lignin can be treated with phenol in the presence of organic solvents, such as methanol or ethanol, prior to resin synthesis. This process is called phenolation or phenolysis (Effendi et al. 2008). During the phenolation process, lignin is thermally treated with phenol in an acidic medium, and this leads to the condensation of phenol with the lignin aromatic ring and side chain. Ether bonds are also cleaved during the process, which decreases the molecular weight of the lignin molecule. The resulting material can react with formaldehyde under either alkaline or acidic conditions to synthesize resol or novolac resins (Ysbrandy et al.1992; Cetin et al. 2002). A typical phenolation process is as follows: lignin is added to a mixture of phenol/ethanol solution. Phenolysis of lignin is allowed to proceed at $70{ }^{\circ} \mathrm{C}$ for a few hours. Ethanol is evaporated subsequently, and a homogenous mixture of lignin and phenol is then obtained. The viscosity of phenolated lignin varies depending on the phenol to lignin ratio. The mixture then is reacted with formaldehyde to synthesize PF resin. Particleboards were prepared using these resins with up to $30 \%$ phenol substituted by phenolated lignin, and they showed similar physical and mechanical properties with those made from commercial PF resins (Cetin et al. 2002).

Phenolation is one of the most used modification methods for lignosulfonate, and the purpose of this method is to increase the content of phenolic hydroxyl groups and to simplify the structure of lignosulfonate. Zhao (2000) reported that the content of phenolic hydroxy groups of lignosulfonate could be increased significantly by treating lignosulfo- 
nate with $\mathrm{m}$-cresol as the phenolation agent and sulphuric acid as the catalyst. Phenolation was conducted by mixing $2 \mathrm{~g}$ of lignosulfonate, $21 \mathrm{~mL}$ of m-cresol, and $67 \mathrm{~g}$ of $\mathrm{H}_{2} \mathrm{SO}_{4}(72 \%)$, followed by stirring the mixture violently at room temperature for $1 \mathrm{~h}$. Sulfonic acid group in lignosulfonate could be cleaved easily under the above reaction conditions. Results from IR, UV, and ${ }^{1} \mathrm{H}$ NMR analysis indicated an increase in the content of phenolic hydroxyl groups in the phenolated lignosulfonate sample. The molecular weight of phenolated lignosulfonate also decreased, which improved the properties of lignosulfonate for the subsequent polymerization with formaldehyde.

Plywood panels made from 100\% phenolated lignin-phenol-formaldehyde (PLPF) failed to meet the Chinese national standard quality of water resistance. But panels made with $50 / 50$ or $75 / 25 \mathrm{PLPF} /$ commercial PF resin (P301, w/w) blends had much better knife test results than the panels made solely with either PLPF or P301 resin. This synergistic effect may be attributed to the mixed resin having better rheological properties, and thus spreading better on wood flake, in comparison to P301. The mixed resin also had higher solids content than PLPF (Vázquez et al. 1997).

\section{REDUCTION}

The aldehyde and ketone moieties in lignin can be reduced to alcohol by some reducing reagents including lithium aluminum hydride, sodium borohydride $\left(\mathrm{NaBH}_{4}\right)$, or hydrogen over a catalyst, such as Raney nickel in an aqueous-organic solvent mixture. Lithium aluminum hydride is a strong but selective hydrogenating agent that can reduce aldehyde, ketone, acid, and ester groups to alcohol without reacting with carbon-carbon bonds in the lignin. It can react with lignin in anhydrous ether at room to elevated temperatures. Sodium borohydride is a weaker hydrogenating agent and only reduces aldehydes and ketones to alcohol. It likewise can react with lignin in an alkaline solution at room to elevated temperatures (Meister 2002). A study by Li et al. (2004) showed that a palladium $(\mathrm{Pd}) /$ carbon catalyst could increase the activity of alkaline lignin. In addition, the catalyst could be reused up to three times without losing any catalytic activity. The modified lignin resulted in the increasing of total hydroxyls, phenolic hydroxyls, and aliphatic hydroxyls by $46.95 \%, 33.74 \%$, and $63.93 \%$, respectively, compared to their original values.

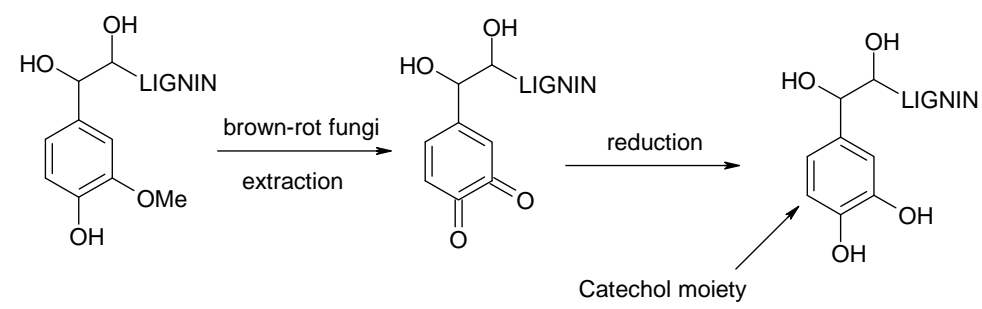

Fig. 1. Scheme of formation of catechol moiety in demethylated lignin (Li et al. 2005)

Li et al. (2005) also used $\mathrm{NaBH}_{4}$ to reduce brown-rot fungi demethylated lignin, and the resulting materials were mixed with polyethylenimine to synthesize a formaldehyde-free, strong, and water-resistant wood adhesive. Lignin could be partially dimethyl- 
ated by brown-rot fungi, followed by reduction with $\mathrm{NaBH}_{4}$ to form catechol moieties (Fig. 1), which are known to have higher reactivity than phenol.

Sun (2009) conducted research on the application of brown-rot fungi decomposed lignin as a wood adhesive. Lignin was first extracted with $1 \% \mathrm{NaOH}$ solution, and the extractant was reduced with $\mathrm{NaBH}_{4}$. The contents of aliphatic hydroxyl and total hydroxyl groups increased to $17.26 \%$ and $21.09 \%$, respectively. Sun also used Raney nickel instead of $\mathrm{NaBH}_{4}$ in the reduction of the brown-rot fungi demethylated lignin in a closed system at $260{ }^{\circ} \mathrm{C}$ and $280{ }^{\circ} \mathrm{C}$ with tetralin as the hydrogen donor. The results showed that the contents of phenolic hydroxyl and total hydroxyl could reach $4.18 \%$ and $17.4 \%$, respectively.

\section{OXIDATION}

Lignin oxidation has been well studied as a means to obtain various chemicals, such as vanillin, syringaldehyde, and 4-hydroxybenzaldehyde (Hatakeyama et al. 1975). Marton et al. (1963) dissolved alkaline lignin in a warm acetic acid solution (90\%). The oxidative agent, $\mathrm{NaIO}_{4}$, was then added to the solution and mixed rapidly. The reaction was stopped quickly by adding a reducing agent. The reaction could also be controlled by removing the reaction system from heat. $\mathrm{NaIO}_{4}$ first oxidized methoxyl groups to quinone groups, and then reducing agent, such as $\mathrm{SO}_{2}$, reduced the quinone to hydroxyl groups.

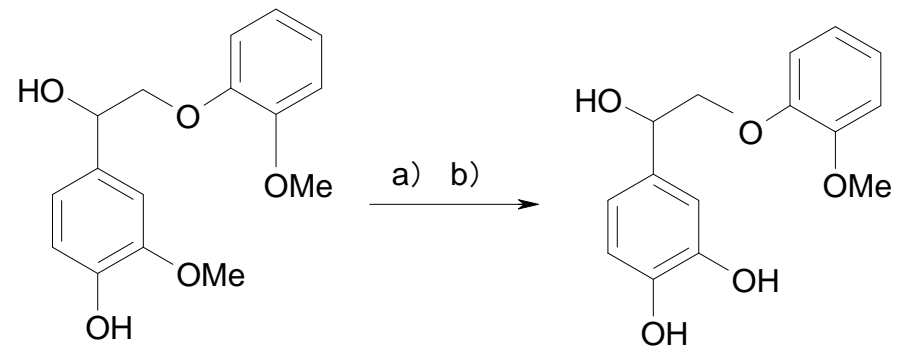

a) IBX, DMC, $\mathrm{H} 2 \mathrm{O} ;$ b) $\mathrm{Na}_{2} \mathrm{~S}_{2} \mathrm{O}_{4}$

Fig. 2. Scheme of oxidation and in situ reduction of neolignan to form catechol moiety (Bernini et al. 2009)

Okamoto et al. (1996) found that in the presence of a catalyst consisting of a metal-porphrin complex and a reductant, anisole could be converted to phenol by molecular oxygen at room temperature with a total turnover of the catalyst about 70 times in $24 \mathrm{~h}$. Potassium dichromate was used to demethylate lignin and thiolignin in the presence of acetic acid (Nagieb et al. 1985; Olivares et al. 1988). The formation of catechol groups in the resulting lignin was reported. The concentration of methoxyl groups decreased from $11.9 \%$ to $2.8 \%$, and the phenolic hydroxyl groups increased from $2.4 \%$ to $11.2 \%$. Alternatively, 2-iodoxybenzoic acid (IBX) was used as a primary oxidant to demethylate lignin in a dimethyl carborate (DMC)/ $\mathrm{H}_{2} \mathrm{O}$ mixture (Bernini et al. 2009). It mimicked the metabolic activity of the cytochrome p-450 enzyme in the de-Omethylation of guaiacyl lignan. The guaiacyl lignan can be converted to the 
corresponding methyl-ester if it has a free carboxylic moiety. The reaction could be conducted under mild conditions. As shown in Fig. 2, lignan was first treated with a small excess of IBX in $\mathrm{H}_{2} \mathrm{O} / \mathrm{DMC}(1 / 9, \mathrm{v} / \mathrm{v})$ solution at room temperature for 3 to $5 \mathrm{~h}$. Then the reaction was quenched by adding some water, followed by an in situ reduction with $\mathrm{Na}_{2} \mathrm{~S}_{2} \mathrm{O}_{4}$ for several minutes until a color change in the solution was observed.

Lignin could also be oxidated by nitrobenzene and copper (II) oxide in an alkaline medium to obtain phenolic compounds, such as syringaldehyde, vanillin, syringic acid, and vanillic acid, in a Burton-Coblin autoclave under $\mathrm{N}_{2}$ environment at 160 to $190{ }^{\circ} \mathrm{C}$ (Villar et al. 1997). Monomeric composition could be obtained using alkaline nitrobenzene (Billa et al. 1996). Significant variations in obtaining different types of aromatic aldehydes as well as hydroxycinnamic acids were found, depending on the changes in the temperature and time of the reaction.

Hydrogen peroxide $\left(\mathrm{H}_{2} \mathrm{O}_{2}\right)$ is a common oxidative bleaching reagent used in pulping and papermaking. There has been a great deal of interest in the reactions of $\mathrm{H}_{2} \mathrm{O}_{2}$ with chromophoric and other reactive groups in lignin. However, the reactions of $\mathrm{H}_{2} \mathrm{O}_{2}$ with lignin are not restricted to chromophoric groups. It can also degrade and solubilize lignin. Decomposition of $\mathrm{H}_{2} \mathrm{O}_{2}$ forms molecular oxygen and other radical species (e.g. $. \mathrm{OH}$ and. $\mathrm{OOH})$, which in turn may react with lignin in a variety of ways, making the total oxidation reaction mechanism extremely complicated (Xiang et al. 2000). The decomposition rate of $\mathrm{H}_{2} \mathrm{O}_{2}$ increases with the increase of temperature and $\mathrm{pH}$ (Reichert et al.1 944). The reaction is also sensitive to trace of transition metal ions, particularly iron, copper, and manganese (Brown et al. 1995). Gierer et al. (1977) used $\beta$-hydroxyarylglycerol- $\beta$-aryl ether (GG) as a lignin model compound to study its degradation by oxygen in an alkaline media. Three reaction pathways were proposed to explain the degradation of GG under different conditions. Two of them involves the oxidation elimination of the side chain by oxygen and $\mathrm{H}_{2} \mathrm{O}_{2}$. The other entails the oxidative cleavage of the $\mathrm{C} \alpha-\mathrm{C} \beta$ bond by oxygen.

\section{HYDROLYSIS}

The phenyl-propane units in lignin are sources of phenolic compounds. Hydrolysis can depolymerize lignin by tearing off the ether that binds the phenyl-propane units together, thus giving rise to low-molecular-mass phenolic compounds. Water at near- and super-critical conditions is a unique solvent for degradation of swelled biomass and solid waste polymers. Degradation of lignin in near- and super-critical water was studied using a batch type reactor (Wahyudiono et al. 2008). The main products in the methanolsoluble fraction were catechol $(28.37 \mathrm{wt} \%)$, phenol $(7.53 \mathrm{wt} \%), m, p$-cresol $(7.87 \mathrm{wt} \%)$, and $o$-cresol $(3.80 \mathrm{wt} \%)$. Alkaline hydrolysis of industrial lignin was able to produce reactive degradation products that could be used to synthesize phenlolic resins. The phenolic hydroxyl content of the hydrolyzed lignin increased when the severity of the treatment increased, and therefore improved the reactivity of lignin toward formaldehyde (Mansouri et al. 2006). Technical lignin (e.g., kraft lignin and lignosulfonate) could be hydrolyzed to smaller molecules after treating with a $5 \% \mathrm{NaOH}$ solution at $180{ }^{\circ} \mathrm{C}$ for 6 h. The resulting compounds were mainly 2-methoxyphenol, 2,6-hydroxy-3,5- 
dimethoxybenzaldehyde (Nekova et al. 2008), etc. Benign et al. (1971) and co-workers proposed hydrolysis of alkaline lignin to monomeric phenols under neutral conditions. They argued that alkali and alkali sulfide did cleave phenolic ethers. However, the alkaline condition would promote the retrogradation and condensation among degraded lignin compounds. Li et al. (2007) proposed that during the alkaline hydrolysis of lignin, depolymerization and repolymerization could pass through the same intermediate state. The recombination of carbon-centered radicals could lead to the formation of strong carbon-carbon bonds and thus result in coke and tar. Other studies also reported the recondensation reaction during the hydrolysis of lignin. Sun (2009) used 1\% $\mathrm{NaOH}$ solution to extract lignin from brown-rot fungi degraded wood and found that the average molecular weight $\left(M_{n}\right)$, weight average molecular weight $\left(M_{w}\right)$, peak molecular weight $\left(M_{p}\right)$, and polydispersity of the lignin increased significantly after reduction with $\mathrm{NaBH}_{4}$, indicating that the recondensation reaction occurred. To overcome this problem, boric acid was used to cap the phenolic hydroxyl groups by forming strongly coordinated boric acid esters with phenolic hydroxyl group, which could block the addition and condensation reactions (Roberts 2008). The composition of the resulting product can be shifted to monomeric compounds by the addition of $\mathrm{NaOH}$ at appropriate concentration. Zhen (2008) found that phenol can inhibit the char formation during both the pyrolysis and hydrolysis of lignin in water/phenol solution at $600{ }^{\circ} \mathrm{C}$.

\section{CONCLUSIONS}

Interest in the utilization of lignin as a substitute for phenol in phenolic resin and for other valuable phenolic compounds has been motivated by the large quantity of lignin-containing biomass, particularly when it is available as a low-cost by-product of the pulping process. Interest has been further prompted by the relatively high price of phenol and more recently by environmental considerations.

Because lignin has less reactive sites than phenol itself, a tremendous amount of research work has been conducted to modify lignin to improve its reactivity. As reviewed in the present work, variations of process and recovery conditions have been investigated to obtain rich phenolic compounds with higher reactivity at low cost and in an environmentally friendly manner. However, no method found to date could meet both criteria. For instance, some methods involving mild reaction conditions need to use organic solvents (e.g., dioxane, acetic acid, or pyridine), which are not environmentally friendly. Lignin oxidation with $\mathrm{H}_{2} \mathrm{O}_{2}$ could be a good choice to improve the properties of lignin, noting that $\mathrm{H}_{2} \mathrm{O}$ is the terminal product. At the same time, an alkaline aqueous solution is a better reaction medium than acidic or neutral environments, in terms of the subsequent synthesis of phenolic resin.

Nevertheless, lignin is still considered the most promising substitute for phenolic compounds due to its readily availability and low cost. For this reason one can expect that efforts will be continued on the commercialization of phenolic compounds derived from waste lignins from the pulp and paper industry. 


\section{ACKNOWLEDGMENTS}

The authors are grateful for the support of the Forestry Public Sector Research

Fund of State Forestry Administration of China (Grant No. 201104004), and Chinese National Forestry Bureau Extention Project (Grant. No. [2010]05).

\section{REFERENCES CITED}

Alonso, M. V., Oliet, M., Rodríguez, F., Astarloa, G., and Echeverría, J. M. (2004). "Use of a methylolated softwood ammonium lignosulfonate as partial substitute of phenol in resol resins manufacture," Journal of Applied Polymer Science 94, 643650.

An, X. N., Schroeder, H. A., and Thompson, G. E. (1995). "Demethylated kraft lignin as a substitute for phenol in wood adhesive," Chemistry and Industry of Forest Products 15(3), 36-42.

Argyropoulos, D. S. and Menachem, S. B. (1997). "Lignin," Advances in Biochemical Engineering/Biotechnology 57, 127-158.

Benign, J. D., and Goldstein, I. S. (1971). "Neutral hydrolysis of alkali lignin to monomeric phenols," Journal of Polymer Science: Part C 36, 467-475.

Bernini, R., Barontini, M., Mosesso, P., Pepe, G., and Willför, S. M. ( 2009). “A selective de-O-methylation of guaiacyl lignans to corresponding catechol derivatives by 2iodoxybenzoic acid (IBX). The role of the catechol moiety on the toxicity of lignans," Organic \& Biomolecular Chemistry 7, 2367-2377.

Billa, E., Tollier, M. T., and Monties, B. (1996). "Characterisation of the monomeric composition of in situ wheat straw lignins by alkaliie nitrobenzene oxidation: Effect of temperature - and reaction time," Journal of the Science of Food and Agriculture $72,250-256$.

Brown, D. G., and Abbot, J. (1995). "Effects of metal ions and stabilisers on peroxide decomposition during bleaching," Journal of Wood Chemistry and Technology 15(1), 85-111.

Campion, S. H., and Suckling, I. D. (1998). "Effect of lignin demethylation on oxygen delignification," Appita Journal 51, 209-212

Cetin, N. S., Özmen, N. ( 2002). "Use of organosolv lignin in phenol-formaldehyde resins for particalboard production-I. Organosolv lignin modified resins," International Journal of Adhension \& Adhensives 22, 477-480.

Effendi, A., Gerhauser, H., and Bridgwater, A. V. ( 2008). "Production of renewable phenolic resins by thermochemical conversion of biomass: A review," Renewable and Sustainable Energy Reviews 12, 2092-2116.

Gierer, J., Imsgard, F., and Noren, I. (1977). "Studies on the degradation of phenolic lignin units of the $\beta$-aryl ether type with oxygen in alkaline media," Acta Chemica Scandinavica B 31, 561-572.

Gosselink, R. J. A., Guran, B., and Abächerli, A. (2004). "Co-ordination network for lignin-standardisation, production and applications adapted to market requirements (Eurolignin)," Industrial Crops and Products 20, 121-129. 
Hatakeyama, H., Iwashita, K., Meshitsuka, G., and Nakano, J. (1975). "Effect of molecular weight on glass transition temperature of lignin," Mokuzai Gakkaishi 21(11), 618-623.

Lee, S. H., Teramoto, Y., and Shiraishi, N. (2002). "Resol-type phenolic resin from liquefied phenolated wood and its application to phenolic foam," Journal of Applied Polymer Science 84, 468-472.

Li, J., Henriksson, G., and Gellerstedt, G. (2007). "Lignin depolymerization/ repolymerization and its critical role for delignification of aspen wood by steam explosion," Bioresource Technology 98, 3061-3068.

Li, K. C., and Geng, X. L. ( 2005). "Formaldehyde-free wood adhesives from decayed wood," Macromol. Rapid Commun. 26, 529-532.

Li, L. Y., Fang, G. Z., and Ren, S. X.(2004). "Alkali lignin activated by Pd/C catalyst," Transactions of China Pulp and Paper 19(2), 129-133.

Lin, Z. X., Ouyang, X. P., Yang, D. J., Deng, Y. H., and Qiu, X. Q. (2010). "Effect of hydroxymethylation of lignin on the properties of lignin-phenol-formaldehyde resins," World Sci-Tech R\&D 32(3), 348-351.

Lora, J. H., and Glasser, W. G. (2002). "Recent application of lignin: A sustainable alternative to nonrenewable materials," Journal of Polymers and the Environment 10, 39-48.

Malutan, T., Nicu, R., and Popa, V. I. (2008). "Contribution to the study of hydroxymethylation reaction of alkali lignin," BioResources 3(1), 13-20.

Mansouri, N. E., Farriol, X., and Salvadó, J. (2006). "Structural modification and characterization of lignosulfonate by a reaction in an alkaline medium for its incorporation into phenolic resins," Journal of Applied Polymer Science102, 32863292.

Mansouri, N. E, and Salvadó, J.(2006). "Structure characterization of technical lignins for the production of adhesives: Application to lignosulfonate, kraft, soda-anthraquinone, organosolv and ethanol process lignins," Industrial Crops and Products 24, 8-16.

Marton, J., Charleston, S. C., and Adler, E. (1963). "Oxidative demethylation of lignin," U.S. 3071570.

Marton, J., Marton, T., Falkchag, S. I., and Adler, E. (1996). "Alkali-catalyzed reactions of formaldehyde with lignins," In: Lignin Structure and Reactions, Marton, J. (ed.), Advances in Chemistry Series 59, American Chemical Society, Washington, D.C., 125-144.

Meister, J. J. ( 2002). "Modification of lignin," J. Macromol. SCI. -Polymer Reviews C42(2), 235-289.

Mu, Y. B., Wang, C. P., Zhao, L. W., and Chu, F. X. ( 2009). "Study on composite adhesive of hydroxymethylated lignosulfonate/pheno1-formaldehyde resin with low free formaldehyde," Chemistry and Industry of Forest Products 29(3), 38-42.

Nagieb, Z. A., and Egypt, C. (1985). "Demethylation of thiolignin by reaction with potassium dichromate-a kinetic study," Wood Sci.Technol. 19, 233-242.

Nekova, S., Vasileva, T., and Stanulov, K. (2008). "Production of phenol compounds by alkaline treatment of technical hydrolysis lignin and wood biomass," Chemistry of Natural Compounds 44(2), 182-185. 
Okamoto, T., Takeda, H., Funabiki, T., Takatani M., and Hamada, R. (1996). "Fundamental studies on the development of lignin-based adhensives. I. Catalytic demethylation of anisole with molecular oxygen," React React. Kinet. Catal. Lett. 58(2), 237-242.

Olivares, M., Guzmán J. A., Natho, A., and Saavedra, A. ( 1988). “Kraft lignin utilization in adhensives," Wood Sci.Technol. 22, 157-165.

Pizzi, A., Cameron, F. A., and Klashorst, G. H. (1989). "Soda bagasse lignin adhesive for particleboard," In: Adhesives from Renewable Resources, ACS Symposium Series 385, Washington, D.C., 82.

Reichert, J. S., Cambell, D. J., and Mills, R. T. (1944). "Sodium per-oxide bleaching of mechanical pulps," Tech. Assoc. Papers 27, 364-370.

Roberts, V. M. (2008). "Homogeneous and heterogeneous catalyzed hydrolysis of lignin," Doctoral Dissertation, Technische Universität München, Munich.

Sun, Q. N. (2009). "Research on preparation of non-formaldehyde adhesive by modified brown-rotted wood," Master Dissertation, Chinese Academy of Forestry, Beijing.

Vázquez, G., González, J., and Antorrena, F. G. (1997). "Effect of chemical modification of lignin on the gluebond performance of lignin-phenolic resins," Bioresource Technology 60, 191-198.

Villar, J. C., Caperos, A., and García-Ochoa, F. (1997). "Oxidation of hardwood kraftlignin to phenolic derivatives nitrobenzene and copper oxide as oxidants," Journal of Wood Chemistry and Technology 17(3), 259-285.

Wahyudiono, Sasaki, M., and Goto, M. (2008). "Recovery of phenolic compounds through the decomposition of lignin in near and supercritical water," Chemical Engineering and Processing 47, 1609-1619.

Wu, S. B., and Zhan, H. Y. (2001). "Characteristics of demethylated wheat straw soda lignin and its utilization in lignin-based phenolic formaldehyde resins," Cellulose Chemistry and Technology 35, 253-262.

Xiang, Q., and Lee, Y. Y. (2000). "Oxidative cracking of precipitated hardwood lignin by hydrogen peroxide," Applied Biochemistry and Biotechnology 84-86, 153-162.

Yang, L. S., and Hightstown, N.J. (1981). "Demethylated lignin and process," U.S. Patent 4,250,088.

Ysbrandy, R. E., Sanderson, R. D., and Gerischer, G. F. R. (1992). “Adhesives from autohydrolysis bagasse lignin, A renewable resource. Part I: The physical properties of laminates made with phenolated lignin novolacs," Holzforschung 46(3), 249-252.

Zhao, B.Y., Hu, K.A., and Wu, R. J. (2000). "Primary study on the phenolic modification of sodium lignosulphoate," Polymer Materials Science and Engineering 1, 158-161.

Zhao, L. W., Griggs, B. F., Chen, C. L., and Hse, C. Y. (1994). "Utilization of softwood kraft lignin as adhensive for the manufacture of reconstituted wood," Journal of Wood Chemistry and Technology 14(1), 127-145.

Zhen, F., Sato, T., Richard, L., and Smith, J. (2008). "Reaction chemistry and phase behavior of lignin in high-temperature and supercritical water," Bioresource Technology 99, 3424-3430.

Article submitted: March 2, 2011; Peer review completed: April 17, 2011; Revised version received and accepted: May 15, 2011; Published: May 17, 2011. 